\title{
Ramsay hunt syndrome - a diagnostic dilemma
}

\begin{abstract}
Ramsay Hunt syndrome is peripheral facial nerve palsy accompanied by an erythematous vesicular rash on the ear (zoster oticus) or in the mouth. Ramsay Hunt Syndrome (RHS) is a rare, severe complication of varicella zoster virus (VZV) reactivation in the geniculate ganglion. Ramsay Hunt syndrome is rare in children and affects both sexes equally. Incidence and clinical severity increases when host immunity is compromised. It is also accompanied by other frequent symptoms like tinnitus, hearing loss, nausea, vomiting, vertigo, and nystagmus due to the close proximity of the geniculate ganglion to the vestibulocochlear nerve within the bony facial canal. Because these symptoms do not always present at the onset, this syndrome can be misdiagnosed. Early and correct treatment should be performed to avoid complications, such as permanent facial nerve dysfunction.
\end{abstract}

We describe a 23year old female who was initially misdiagnosed as Bell's palsy and later developed vesicles on the pinna which led to a diagnosis of RHS and successfully treated with acyclovir and steroids.

Keywords: facial palsy, ramsay hunt syndrome, varicella zoster, herpes zoste
Volume 8 Issue I - 2017

Pirabu Sakthivel, Chirom Amit Singh, Smriti

Panda, Suresh K, Subagar Anbarasan

Department of Otorhinolaryngology Head and Neck surgery

All India Institute of Medical Sciences, New Delhi, India

Correspondence: Pirabu Sakthivel Department of

Otorhinolaryngology and Head Neck surgery All India Institute of Medical Sciences New Delhi I I0002, India, Tel 9958744547, Email pirabusakthivel@gmail.com

Received: June 01, 2017 | Published: July 17, 2017
Abbreviations: RHS, ramsay hunt syndrome; HZO, herpes zoster oticus; VZV, varicella zoster virusl; PCR, polymerase chain reaction

\section{Introduction}

Ramsay Hunt Syndrome (RHS), also called Herpes Zoster Oticus (HZO), is a rare, severe complication of varicella zoster virus (VZV) reactivation in the geniculate ganglion. The classic triad consists of otalgia, vesicles in the auditory canal and ipsilateral facial paralysis. ${ }^{1}$ Facial palsy, which occurs annually in 30 of 100000 individuals in the general population, may have various causes, ranging from head trauma to an idiopathic episode. ${ }^{2}$ Although RHS is rare, it is the second most common cause of facial paralysis (after Bell's palsy) with non -traumatic peripheral facial paralysis. Early diagnosis and accurate treatment for patients with idiopathic facial palsy (Bell palsy) and Ramsay Hunt syndrome may accelerate recovery and prevent possible complications. Without treatment, full recovery of the facial paralysis occurs in as little as $20 \%$ of cases; this is much improved if treatment is started within 72 hours. $^{3}$ Among the agents most frequently used to treat these conditions are steroids, antiviral agent's vasodilators, and multivitamins. Due to the anti-inflammatory effect of steroids, an enhanced recovery can be achieved in patients with steroid treatment in Bell palsy and Ramsay Hunt syndrome. ${ }^{2}$ In addition, the treatment for patients with Ramsay Hunt with antiviral agents may prevent disease progression and improve prognosis by inhibiting the spread of herpes zoster virus. ${ }^{4,5}$

\section{Case report}

A 23-year-old female complained of Right ear pain for 3days duration; on examination the ear was normal and she was prescribed analgesics. Two days later, the patient noticed weakness on the entire right side of the face. She was diagnosed with Bell's palsy and oral treatment with methylprednisolone $(1 \mathrm{mg} / \mathrm{kg} /$ day) was commenced. On the second day of treatment with steroids, painful blisters with crusts developed in her right ear (Figure 1A). There was no history of dizziness or tinnitus. The patient had Grade 2 House- Brackmann (HB) facial paralysis (Figure 1B, 1C). Laboratory findings (complete blood count and erythrocyte sedimentation rate) were within normal limits. The patient had a normal pure tone audiogram and impedance audiometry. Based on the above finding, she was diagnosed with RHS and a 7-day course of oral acyclovir $800 \mathrm{mg} 5$ times a day was commenced. The patient had no other comorbidities. On follow up, the ear lesions disappeared within 2 weeks, and the facial paralysis resolved completely by the end of 4 weeks.

\section{Discussion}

RHS or HZO is characterized by facial paralysis, ear pain, and erythematous vesicular lesions in the ear and oral mucosa. Herpes zoster is seen as a disease of older people, and incidence and severity increases with age. ${ }^{6}$ The VZV reactivation in the geniculate ganglia and subsequent neural inflammation, pressure, and possible destruction of the facial nerve in the temporal bone are suspected to cause facial palsy, while VZV migrates from the geniculate ganglia into the skin around the ear or into the oropharynx via the sensory fibers, where it replicates and produces zoster in RHS. ${ }^{7}$ Frequently, there is involvement of VIII cranial nerve producing hearing impairment and vertigo. Involvement of cranial nerves V, IX, X, XI, and XII occurs less frequently. RHS, which develops in $1 \%$ of patients with a herpes zoster infection, was first described by Ramsay Hunt in 1907.7 The primary etiologic agent of RHS is VZV but Bell's palsy, in contrast, has been attributed to herpes simplex virus type- $1 .{ }^{8}$

RHS commences with the classical prodromal symptoms of pain, fever, and fatigue, 1-3days in duration. Next, herpetic vesicles begin to develop in the external auditory canal, tympanic membrane, and/ or the anterior two-thirds of the tongue. Facial paralysis usually develops within 1-2weeks after the rash appears. ${ }^{2}$ Facial palsy and zoster do not always appear simultaneously, and some patients with RHS exhibit facial palsy several days before as in our case or after the onset of zoster. ${ }^{8}$ VZV also causes acute peripheral facial palsy with the absence of skin lesions; such cases are termed zoster sine herpete and are usually diagnosed using serological assays or polymerase chain reaction $(\mathrm{PCR}) .{ }^{8,9}$

Various associated complications include corneal abrasions and ulcers, if eye-lid closure is impaired, secondary infection with bacteria (cellulitis), Post herpetic neuralgia, as with other Varicella Zoster virus reactivations, permanent facial paralysis, and long term ipsilateral hearing loss and tinnitus. ${ }^{10}$ 
Bell's palsy is a diagnosis of exclusion for unilateral facia weakness. Painful erythematous vesicular rash suggest RHS. The ear pain in RHS can be mistaken for otitis. Onset of rash and development of facial palsy differentiate RHS from otitis. Trigeminal neuralgia pain tends to be paroxysmal, stimulated by triggers and it is not associated with any skin manifestations or neurological losses differentiating it from RHS. ${ }^{10}$

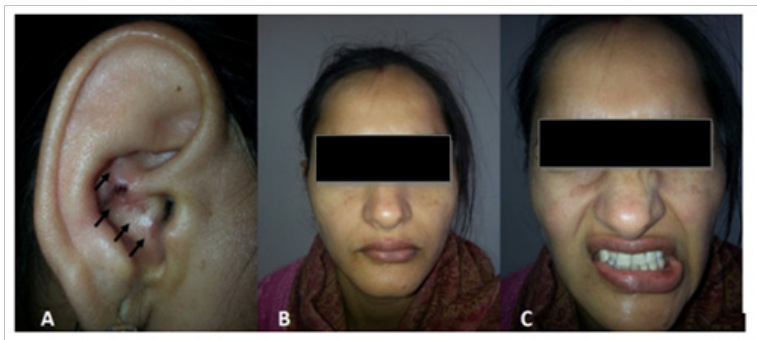

Figure I A) Vesicles with crusts seen on the pinna (black arrows); B) Facial symmetry at rest ;C) clinical picture showing Grade 2 house brackmann weakness (complete eye closure with minimal asymmetry and weakness on the right side).

The most recommended therapy for RHS is the combination of acyclovir and prednisone. ${ }^{3}$ Acyclovir is an effective antimicrobial agent against actively replicating herpes zoster viruses. Acyclovir itself is not active. It must first be phosphorylated by viral thymidine kinase to form a triphosphate. Acyclovir triphosphate inhibits viral DNA polymerase and, thus, DNA replication. Importantly, no statistically significant outcome differences were noted between patients treated with intravenous or oral acyclovir. Because of increasing viral resistance to acyclovir, newer drugs, such as valacyclovir, famciclovir, penciclovir and brivudine, are being more commonly used. ${ }^{8}$

Adjunctive steroid therapy can be helpful in the management of the facial paralysis of RHS. ${ }^{11}$ A study on 80 RHS patients with different levels of severity treated with acyclovir-prednisone combination showed complete facial recovery, i.e., House grade I, in $52 \%$ patients, no matter what their pre-treatment gradings were. ${ }^{3}$ Methylprednisolone has a higher affinity to glucocorticoid receptors, and a higher anti-inflammatory action when compared with the prednisone, prednisolone, or hydrocortisone. ${ }^{11}$

Advanced age, initial House-Brackmann grades V or more, time before commencement of treatment, associated metabolic diseases, impairment of the cochleovestibular, or other cranial nerves, oropharynx lesions, dry eye, and lagophthalmus must be assessed at the initial physical examination, since they suggest worse prognosis of the facial palsy secondary to Ramsay Hunt syndrome. ${ }^{2,11}$

To conclude, although patients with Ramsay Hunt syndrome have poorer prognosis than do those with Bell's palsy, early combination therapy of steroids with antivirals within 3days of the onset of facial palsy is beneficial.

\section{Conflict of interest statement}

The authors report no conflict of interest.

\section{Source of Funding}

None.

\section{Acknowledgments}

None.

\section{References}

1. Adour KK. Otological complications of herpes zoster. Ann Neurol. 1994;35(Suppl 1):S62-S64.

2. Ryu EW, Lee HY, Lee SY, et al. Clinical manifestations and prognosis of patients with Ramsay Hunt syndrome. Am J Otolaryngol. 2012;33(3):313-318.

3. Murakami S, Hato N, Horiuchi J, et al. Treatment of Ramsay hunt syndrome with acyclovir-prednisone: significance of early diagnosis and treatment. Ann Neurol. 1997;41(3):353-357.

4. Campbell KE, Brundage JF. Effects of climate, latitude, and season on the incidence of Bell's palsy in the US Armed Forces, October 1997 to September 1999. Am J Epidemiol. 2002;156:32-39.

5. Peitersen E. Bell's palsy: the spontaneous course of 2,500 peripheral facial nerve palsies of different etiologies. Acta Otolaryngol Suppl. 2002;549:4-30.

6. Burke BL, Steele RW, Beard OW, et al. Immune responses to varicellazoster in the aged. Arch Intern Med. 1982;142(2):291-293.

7. Hunt JR. On herpetic inflammation of the geniculate ganglion. A new syndrome and its complications. J Nerv Ment Dis. 1907;34:73-96.

8. Gondivkar S, Parikh V, Parikh R. Herpes zoster oticus: A rare clinical entity. Contemporary Clinical Dentistry. 2010;1(2):127-129.

9. Murakami S, Honda N, Mizobuchi M, et al. Rapid diagnosis of varicella zoster virus infection in acute facial palsy. Neurology. 1998;51(4):1202-1205.

10. Worme M, Chada R, Lavallee L. An unexpected case of Ramsay Hunt syndrome: case report and literature review. BMC Res Notes. 2013;6:337.

11. Monsanto RD, Bittencourt AG, Bobato Neto NJ, et al. Treatment and Prognosis of Facial Palsy on Ramsay Hunt Syndrome: Results Based on a Review of the Literature. Int Arch Otorhinolaryngol. 2016;20(4):394-400. 